\title{
THE NONEXISTENCE OF CERTAIN IDENTITIES IN THE THEORY OF PARTITIONS AND COMPOSITIONS
}

\author{
HENRY L. ALDER
}

1. Introduction. Let $q_{d, m}(n)$ be the number of partitions of $n$ into parts differing by at least $d$, each part being greater than or equal to $m$. We discuss here the question of the existence of identities involving $q_{d, m}(n)$ analogous to the tautology:

$$
\sum_{n=0}^{\infty} q_{1,1}(n) x^{n}=\prod_{\nu=1}^{\infty}\left(1+x^{\nu}\right)
$$

the Euler identity:

$$
\sum_{n=0}^{\infty} q_{1,1}(n) x^{n}=\prod_{v=1}^{\infty} \frac{1}{1-x^{2 p-1}},
$$

and the Rogers-Ramanujan identities:

$$
\begin{aligned}
& \sum_{n=0}^{\infty} q_{2,1}(n) x^{n}=\prod_{\nu=0}^{\infty} \frac{1}{\left(1-x^{5 v+1}\right)\left(1-x^{5 v+4}\right)}, \\
& \sum_{n=0}^{\infty} q_{2,2}(n) x^{n}=\prod_{\nu=0}^{\infty} \frac{1}{\left(1-x^{5 v+2}\right)\left(1-x^{5 v+3}\right)} .
\end{aligned}
$$

We shall in fact show that, aside from the following simple extensions of the first two:

$$
\begin{aligned}
\sum_{n=0}^{\infty} q_{1, m}(n) x^{n} & =\prod_{\nu=m}^{\infty}\left(1+x^{\nu}\right), \\
\sum_{n=0}^{\infty} q_{1, m}(n) x^{n} & =\frac{\prod_{\nu=m}^{\infty}\left(1-x^{2 \nu}\right)}{\prod_{\nu=m}^{\infty}\left(1-x^{\nu}\right)} \\
& =\frac{1}{\left(1-x^{m}\right)\left(1-x^{m+1}\right) \cdots\left(1-x^{2 m-1}\right)} \prod_{\nu=m}^{\infty} \frac{1}{1-x^{2 \nu+1}},
\end{aligned}
$$

no other such identities exist. More specifically we shall prove the following two theorems, both of which were proved for the case

Presented to the Society, November 29, 1947; received by the editors September 2, 1947. 
$m=1$ by D. H. Lehmer. ${ }^{1}$

THEOREM 1. The number $q_{d, m}(n)$ of partitions of $n$ into parts differing by at least $d$, each part being greater than or equal to $m$, is not equal to the number of partitions of $n$ into parts taken from any set of integers whatsoever unless $d=1$ or $d=2, m=1,2$.

THEOREM 2. The number $q_{d, m}(n)$ of partitions of $n$ into parts differing by at least $d$, each part being greater than or equal to $m$, is not equal to the number of partitions of $n$ into distinct parts taken from any set of integers whatsoever unless $d=1$.

If we now define $Q_{d}(n)$ as the number of partitions of $n$ into parts congruent to 1 or $d+2$ modulo $d+3$, then we have $q_{1,1}(n)=Q_{1}(n)$ by the Euler identity and $q_{2,1}(n)=Q_{2}(n)$ by the first Rogers-Ramanujan identity. A theorem of I. Schur states that $Q_{3}(n)$ is equal to the number of partitions of $n$ into parts differing by 3 or more among which no two consecutive multiples of 3 appear. Thus $q_{3,1}(n) \geqq Q_{3}(n)$, and in fact $q_{3,1}(n)-Q_{3}(n)$ has a rather simple interpretation. Numerical evidence for $d=4,5$ seems to indicate the plausibility of the conjecture that $q_{d, 1}(n) \geqq Q_{d}(n)$ for all positive integers $d$ and $n$. For $d \geqq 4$, however, there seems to be no simple interpretation of the difference $q_{d, 1}(n)-Q_{d}(n)$, even if we could prove it to be non-negative. The following theorem shows in particular that there cannot be an interpretation exactly like that for $d=3$.

THEOREM 3. The number of partitions of $n$ into parts differing by at least $d$ and where parts divisible by $d$ differ by at least $2 d$ is not equal to the number of partitions of $n$ into parts taken from any set of integers whatsoever if $d>3$. 1.

Finally we have for compositions a theorem analogous to Theorem

THEOREM 4. The number $c_{d, m}(n)$ of compositions of $n$ into parts differing by at least $d$, each part being greater than or equal to $m$, is not equal to the number of compositions of $n$ into parts taken from any set of integers whatsoever.

We shall make use of the following two important formulae, which give the generating functions of $q_{d, m}(n)$ and $c_{d, m}(n)$ in a closed form:

${ }^{1}$ D. H. Lehmer, Two nonexistence theorems on partitions, Bull. Amer. Math. Soc vol. 52 (1946) pp. 538-544. See this paper also for references to the sources of the facts quoted here. 


$$
\begin{aligned}
& \sum_{n=0}^{\infty} q_{d, m}(n) x^{n}=\sum_{s=0}^{\infty} \frac{x^{m s+d s(s-1) / 2}}{(1-x)\left(1-x^{2}\right) \cdots\left(1-x^{s}\right)}, \\
& \sum_{n=0}^{\infty} c_{d, m}(n) x^{n}=\sum_{s=0}^{\infty} \frac{s ! x^{m s+d s(s-1) / 2}}{(1-x)\left(1-x^{2}\right) \cdots\left(1-x^{s}\right)} .
\end{aligned}
$$

The first is proved exactly as Lehmer proved his Theorem 1 (which is the case $m=1$ ), namely by using the equality

$m+(m+d)+(m+2 d)+\cdots+(m+(s-1) d)=m s+d s(s-1) / 2$.

The second follows from the observation that each partition into $s$ parts differing by at least $d$, each part being greater than or equal to $m$, gives rise to $s$ ! compositions.

2. Proof of Theorem 1. We suppose that the theorem is false and that there exists such a set of integers $a_{1}<a_{2}<a_{3}<\ldots$. Then making use of (1) we would have

$$
\begin{aligned}
\prod_{\nu=1}^{\infty}\left(1-x^{a_{\nu}}\right)^{-1} & =\sum_{s=0}^{\infty} \frac{x^{m s+d s(s-1) / 2}}{(1-x)\left(1-x^{2}\right) \cdots\left(1-x^{s}\right)} \\
& =1+\frac{x^{m}}{1-x}+\frac{x^{2 m+d}}{(1-x)\left(1-x^{2}\right)}+\cdots .
\end{aligned}
$$

Therefore $a_{1}=m$. Multiplying (3) by $1-x^{m}$, we have:

$$
\begin{aligned}
\prod_{\nu=2}^{\infty}\left(1-x^{a_{\nu}}\right)^{-1}= & +x^{m+1}+x^{m+2}+\cdots+x^{2 m-1} \\
& +\frac{x^{2 m+d}\left(1-x^{m}\right)}{(1-x)\left(1-x^{2}\right)}+\cdots
\end{aligned}
$$

Now $a_{2}=m+1$. Multiplying (4) by $1-x^{m+1}$ we have:

$$
\begin{aligned}
\prod_{\nu=3}^{\infty}\left(1-x^{a_{\nu}}\right)^{-1}= & 1+x^{m+2}+x^{m+3}+\cdots \\
& +x^{2 m-1}-x^{2 m+2}-x^{2 m+3}-x^{2 m+4}-\cdots \\
& +\frac{x^{2 m+d}\left(1-x^{m}\right)\left(1-x^{m+1}\right)}{(1-x)\left(1-x^{2}\right)}+\cdots
\end{aligned}
$$

Noting that for $d \geqq 3$ the coefficient of $x^{2 m+2}$ on the right-hand side of (5) is negative we have a contradiction, as the coefficients of the terms on the left-hand side are all non-negative. It remains to prove the theorem for $d=2$. Here we can suppose that $m \geqq 3$, as we know the 
cases $m=1$ and $m=2$ from the Rogers-Ramanujan identities. If $d=2$, equation (5) can be written for odd values of $m$ in the form:

$$
\begin{aligned}
& \prod_{\nu=3}^{\infty}\left(1-x^{\left.a_{\nu}\right)^{-1}=}+1\right. \\
& +x^{m+2}+x^{m+3}+\cdots \\
& +x^{2 m-1}+x^{2 m+4}+x^{2 m+5}+2 x^{2 m+6}+2 x^{2 m+7} \\
& +3 x^{2 m+8}+3 x^{2 m+9}+\cdots \\
& +\left(\left[\frac{c}{2}\right]-1\right) x^{2 m+c}+\cdots \\
& +\left(\frac{m-3}{2}\right) x^{3 m}+\left(\frac{m+1}{2}\right) x^{3 m+1}+\cdots+x^{4 m} \\
& +\frac{\left(x^{3 m+6}+x^{3 m+9}+x^{3 m+12}+\cdots\right)\left(1+x^{2}+x^{4}+\cdots+x^{m-1}\right)}{1-x} \\
& +\frac{x^{4 m+12}+\cdots}{(1-x)\left(1-x^{2}\right)\left(1-x^{3}\right)\left(1-x^{4}\right)}+\cdots
\end{aligned}
$$

Since $a_{3}=m+2, a_{4}=m+3, \cdots, a_{m}=2 m-1$, the left-hand side of (6) can be written as

$$
\begin{aligned}
&\left(1+x^{m+2}+x^{2 m+4}+\cdots\right)\left(1+x^{m+3}+x^{2 m+6}+\cdots\right) \cdots \\
& \cdot\left(1+x^{2 m-1}+x^{4 m-2}+\cdots\right) \prod_{\nu=m+1}^{\infty}\left(1-x^{a_{\nu}}\right)^{-1}
\end{aligned}
$$

Considering only that part of (7) which precedes the product sign, we see that in that part the coefficient of $x^{2 m+c}$ for $4 \leqq c \leqq m$ is equal to $[c / 2]-1$, that is, the same as the coefficient of the corresponding term on the right-hand side of (6). The coefficient of $x^{3 m+1}$ in the part of $(7)$ before the product sign is seen to equal $(m-1) / 2$, whereas the corresponding term on the right-hand side of (6) has as coefficient $(m+1) / 2$. Therefore $a_{m+1}=3 m+1$, and the left-hand side of (6) can now be written as:

$$
\left(1-x^{m+2}\right)^{-1}\left(1-x^{m+3}\right)^{-1} \ldots
$$

$$
\cdot\left(1-x^{2 m-1}\right)^{-1}\left(1-x^{3 m+1}\right)^{-1} \prod_{\nu=m+2}^{\infty}\left(1-x^{a_{\nu}}\right)^{-1} .
$$

We shall now determine the coefficient of $x^{4 m+3}$ on both sides of (6), remembering that the left-hand side of (6) can be written as (8). Denoting the coefficient of $x^{4 m+3}$ in 


$$
\left(1-x^{m+2}\right)^{-1}\left(1-x^{m+3}\right)^{-1}\left(1-x^{m+4}\right)^{-1} \cdots\left(1-x^{2 m-1}\right)^{-1}
$$

by $T_{m}$, we see that $x^{4 m+3}$ is obtained-except for the contribution from (9)-only as $x^{m+2} \cdot x^{3 m+1}$, so that the coefficient of $x^{4 m+3}$ on the left-hand side of (6) is $T_{m}+1$ or $T_{m}+2$, depending on whether one of the $a$ 's is equal to $4 m+3$ or not. To determine $T_{m}$ we note that (9) can be written as

$$
\begin{aligned}
& \left(1+x^{m+2}+x^{2 m+4}+x^{3 m+6}+x^{4 m+8}+\cdots\right) \\
& \cdot\left(1+x^{m+3}+x^{2 m+6}+x^{3 m+9}+\cdots\right) \\
& \cdot\left(1+x^{m+4}+x^{2 m+8}+\cdots\right) \cdots\left(1+x^{2 m-1}+x^{4 m-2}+\cdots\right) .
\end{aligned}
$$

Now $x^{4 m+3}$ can be obtained in three ways:

(a) As the product of $x^{2 m+2 k}$ and $x^{2 m-(2 k-3)}$ with $4 \leqq 2 k \leqq 2 m-2$ and $1 \leqq 2 k-3 \leqq m-2$ and $2 m+2 k \neq 2(2 m-2 k+3)$. This can happen in $(m-1) / 2$ ways if $m \equiv 0(\bmod 3)$ and in $(m-3) / 2$ ways if $m \neq \equiv(\bmod 3)$.

(b) As a third order term $x^{3(m+m / 3+1)}$. This can only happen if $m \equiv 0(\bmod 3)$ exactly once. Therefore cases $(a)$ and $(b)$ together occur $(m-1) / 2$ times whether $m \equiv 0(\bmod 3)$ or not.

(c) As the product of $x^{m+k_{1}}, x^{m+k_{2}}$ and $x^{m+k_{3}}$ with $2 \leqq k_{1}<k_{2}<k_{3}$ $\leqq m-1$. This can happen in

$$
\begin{aligned}
\sum_{2 \leqq k_{1}<k_{2}<k_{3} \leqq m-1, k_{1}+k_{2}+k_{3}=m+3} 1 & =\sum_{k_{1}, 2 \leqq k_{1} \leqq m / 3} \sum_{k_{2}, k_{3}, k_{1}<k_{2}<k_{3}, k_{2}+k_{3}=m+3-k_{1}} 1 \\
& =\sum_{k_{1}=2}^{[m / 3]} \sum_{0<i<j, i+j=m+3-3 k_{1}} 1 \\
& =\sum_{k=2}^{[m / 3]}\left[\frac{m+2-3 k_{1}}{2}\right]
\end{aligned}
$$

ways. (We have used $k_{2}=i+k_{1}, k_{3}=j+k_{1}$.) Consequently:

$$
T_{m}=\sum_{k_{1}=1}^{[m / 3]}\left[\frac{m+2-3 k_{1}}{2}\right]
$$

and the coefficient of $x^{4 m+3}$ on the left-hand side of (6) is equal to:

$$
1+\sum_{k=1}^{[m / 3]}\left[\frac{m+2-3 k}{2}\right] \text { or } 2+\sum_{k=1}^{[m / 3]}\left[\frac{m+2-3 k}{2}\right] .
$$

On the other hand the coefficient of $x^{4 m+3}$ on the right-hand side of (6) appearing only due to the contribution from the term

$$
\frac{\left(x^{3 m+6}+x^{3 m+9}+x^{3 m+12}+\cdots\right)\left(1+x^{2}+x^{4}+\cdots+x^{m-1}\right)}{1-x}
$$


is equal to

$$
\begin{aligned}
0 \leqq 2 j \leqq m-1,6 \leqq k \leqq m+3,2 j+(3 m+k)=4 m+3 & \left(\left[\frac{k}{3}\right]-1\right) \\
= & \sum_{0 \leqq 2 j \leqq m-3}\left(\left[\frac{m+3-2 j}{3}\right]-1\right)=\sum_{0 \leqq j \leqq(m-3) / 2}\left[\frac{m-2 j}{3}\right] \\
= & \sum_{0 \leqq j \leqq(m-3) / 2} 1 \leqq \sum_{11 \leqq(m-2 j) / 3} 1 \\
= & \sum_{k=1}^{[m / 3]}\left[\frac{m+2-3 k}{2}\right] .
\end{aligned}
$$

Therefore the coefficient of $x^{4 m+3}$ on the left-hand side of (6) is larger by at least 1 than the corresponding coefficient on the right-hand side of (6), which is a contradiction.

For even values of $m$ the proof proceeds analogously. Again we have in this case: $a_{1}=m, a_{2}=m+1, a_{3}=m+2, \cdots, a_{m}=2 m-1$. Comparing again the coefficient of $x^{4 m+3}$ we are led to exactly the same expressions as in (10) and (11).

3. Proof of Theorem 2. Again we suppose that the theorem is false, in which case a set consisting of integers $a_{1}<a_{2}<a_{3}<\cdots$ would exist such that

$$
\begin{aligned}
\prod_{\nu=1}^{\infty}\left(1+x^{a_{\nu}}\right)=1 & +\frac{x^{m}}{1-x}+\frac{x^{2 m+d}}{(1-x)\left(1-x^{2}\right)} \\
& +\frac{x^{3 m+3 d}}{(1-x)\left(1-x^{2}\right)\left(1-x^{3}\right)}+\cdots
\end{aligned}
$$

Therefore $a_{1}=m$. Multiplying both sides of (12) by $1-x^{m}$ we have:

$$
\begin{aligned}
& \left(1-x^{2 m}\right) \prod_{\nu=2}^{\infty}\left(1+x^{a_{\nu}}\right)=1+x^{m+1}+x^{m+2}+\cdots \\
& +x^{2 m-1}+\frac{x^{2 m+d}\left(1-x^{m}\right)}{(1-x)\left(1-x^{2}\right)} \\
& \quad+\frac{x^{3 m+3 d}\left(1-x^{m}\right)}{(1-x)\left(1-x^{2}\right)\left(1-x^{3}\right)}+\cdots
\end{aligned}
$$

We note that $a_{2}=m+1, a_{3}=m+2, \cdots, a_{m}=2 m-1$. Now, since $a_{k}+a_{l} \geqq 2 m+3$ for $2 \leqq k<l \leqq m$, we have $a_{m+1}=2 m$, so that (13) can be written: 


$$
\begin{aligned}
& \left(1-x^{4 m}\right)\left(1+x^{m+1}\right)\left(1+x^{m+2}\right) \cdots\left(1+x^{2 m-1}\right) \prod_{\nu=m+2}^{\infty}\left(1+x^{a_{\nu}}\right) \\
& =1+x^{m+1}+x^{m+2}+\cdots+x^{2 m-1} \\
& \quad+\frac{x^{2 m+d}\left(1-x^{m}\right)}{(1-x)\left(1-x^{2}\right)}+\frac{x^{3 m+3 d}\left(1-x^{m}\right)}{(1-x)\left(1-x^{2}\right)\left(1-x^{3}\right)}+\cdots
\end{aligned}
$$

Case 1. $m \geqq 3, d \geqq 4$.

In this case the coefficient of $x^{2 m+3}$ on the right-hand side of (14) is zero, whereas the coefficient of the same term on the left-hand side is greater than or equal to one, so that we have a contradiction.

Case 2. $m \geqq 3, d=3$.

In this case equation (14) reduces to:

$$
\begin{aligned}
& \left(1-x^{4 m}\right)\left(1+x^{m+1}\right)\left(1+x^{m+2}\right) \cdots\left(1+x^{2 m-1}\right) \prod_{\nu=m+2}^{\infty}\left(1+x^{a_{\nu}}\right) \\
& =1+x^{m+1}+x^{m+2}+\cdots+x^{2 m-1} \\
& \quad+\frac{x^{2 m+3}\left(1-x^{m}\right)}{(1-x)\left(1-x^{2}\right)}+\frac{x^{3 m+9}\left(1-x^{m}\right)}{(1-x)\left(1-x^{2}\right)\left(1-x^{3}\right)}+\cdots
\end{aligned}
$$

Comparing now the coefficient of $x^{3 m+2}$ on both sides of (15) we note that on the left-hand side the term $x^{3 m+2}$ is obtained only as the product $x^{k} \cdot x^{l}$ with $m+1 \leqq k<l \leqq 2 m-1$, since $a_{m+2}>2 m+3$ and $x^{k} \cdot x^{l} \cdot x^{n}$ does not equal $x^{3 m+2}$ for $m+1 \leqq k<l<n \leqq 2 m-1$. Therefore the coefficient of $x^{3 m+2}$ on the left-hand side of $(15)$ is $[(m+1) / 2]$ -1 or $[(m+1) / 2]-2$, according as one of the $a$ 's equals $3 m+2$ or not. The coefficient of $x^{3 m+2}$ on the right-hand side of (15), appearing solely as the contribution from the term

$$
\frac{x^{2 m+3}\left(1-x^{m}\right)}{(1-x)\left(1-x^{2}\right)}
$$

can easily be seen to be equal to $[(m+1) / 2]$, again a contradiction.

Case 3. $m \geqq 3, d=2$.

We substitute $d=2$ in (14) and compare, if $m$ is even, just as in case 2 the coefficients of $x^{3 m+2}$ on both sides and find the one on the lefthand side to be $m / 2-1$ or $m / 2-2$ and on the right-hand side $m / 2$. For odd values of $m$, however, we compare the coefficients of $x^{3 m+1}$ and calculate it to be $(m+1) / 2-1$ or $(m+1) / 2-2$ on the left and $(m+1) / 2$ on the right-hand side.

Case 4. $m=2, d \geqq 2$.

Here equation (14) can be written: 


$$
\begin{aligned}
\left(1-x^{8}\right)\left(1+x^{3}\right) & \prod_{\nu=4}^{\infty}\left(1+x^{a_{\nu}}\right) \\
& =1+x^{3}+\frac{x^{4+d}}{1-x}+\frac{x^{6+3 a}}{(1-x)\left(1-x^{3}\right)}+\cdots
\end{aligned}
$$

Now let $\lambda$ be defined by

$$
2^{\lambda-1}<d+4 \leqq 2^{\lambda} .
$$

Since the coefficient of $x^{k}$ on the right of (16) vanishes for $3<k$ $<4+d$, we need: $a_{4}=a^{3}, a_{5}=2^{4}, \cdots, a_{\lambda}=2^{\lambda-1}$, so we can rewrite (16):

$$
\begin{aligned}
\left(1-x^{2^{\lambda}}\right)\left(1+x^{3}\right) & \prod_{\nu=\lambda+1}^{\infty}\left(1+x^{a_{\nu}}\right) \\
& =1+x^{3}+\frac{x^{4+d}}{1-x}+\frac{x^{6+3 d}}{(1-x)\left(1-x^{3}\right)}+\cdots
\end{aligned}
$$

Therefore $a_{\lambda+1}=4+d$. The coefficient of $x^{2^{\lambda}}$ on the right-hand side of (18) is +1 , since $6+3 d>2^{\lambda}$ by (17). If we suppose that $a_{\lambda+j}+3 \neq 2^{\lambda}$ for every $j \geqq 1$, the coefficient of $x^{2^{\lambda}}$ on the left-hand side of (18) is seen to be 0 or -1 , according as some $a_{\lambda+i}$ is equal to $2^{\lambda}$ or not.

For the case where $a_{\lambda+j}+3=2^{\lambda}$ for some $j \geqq 1$, we note that: $a_{\lambda+2}=5+d, a_{\lambda+3}=6+d$. Considering first now the case where $j=1$, that is, $d+7=2^{\lambda}$, we notice that for the smallest value of $d$ satisfying this relation, that is, $d=9$, we have: $a_{6}=14, a_{7}=15, a_{8}=16, a_{9}=19$, $a_{10}=20, a_{11}=21, a_{12}=25$, so that (18) becomes in this case:

$$
\begin{aligned}
& \left(1-x^{32}\right)\left(1+x^{3}\right)\left(1+x^{13}\right)\left(1+x^{14}\right)\left(1+x^{15}\right)\left(1+x^{19}\right)\left(1+x^{20}\right)\left(1+x^{21}\right) \\
& \cdot\left(1+x^{25}\right) \prod_{\nu=13}^{\infty}\left(1+x^{a_{\nu}}\right)=1+x^{3}+\frac{x^{13}}{1-x}+\frac{x^{33}}{(1-x)\left(1-x^{3}\right)}+\cdots,
\end{aligned}
$$

which is impossible, as the coefficient of $x^{28}$ on the left-hand side is greater than or equal to 2 , whereas it is 1 on the right-hand side. We therefore can suppose $d>9$. In that case, as is seen from (18): $a_{\lambda+2}=d+5, a_{\lambda+3}=d+6, a_{\lambda+4}=2^{\lambda}=d+7, a_{\lambda+5}=d+10, a_{\lambda+6}=d+11$, so that (18) now becomes:

$$
\begin{aligned}
& \left(1-x^{2^{\lambda+1}}\right)\left(1+x^{3}\right)\left(1+x^{4+d}\right)\left(1+x^{5+d}\right)\left(1+x^{6+d}\right)\left(1+x^{10+d}\right) \\
& \cdot\left(1+x^{11+d}\right) \prod_{\nu=\lambda+7}^{\infty}\left(1+x^{a_{\nu}}\right) \\
& \quad=1+x^{3}+\frac{x^{4+d}}{1-x}+\frac{x^{6+3 d}}{(1-x)\left(1-x^{3}\right)}+\cdots
\end{aligned}
$$


We note that the coefficient of $x^{2 d+15}$ on the left-hand side of (19) is greater than or equal to 2 , since the term $x^{2 d+15}$ is obtained both as $x^{4+d} \cdot x^{11+d}$ and as $x^{5+d} \cdot x^{10+d}$ and $2^{\lambda+1} \neq 2 d+15$. The coefficient of the same term on the right-hand side, however, is 1 , since $3 d+6>2 d+15$ for $d>9$, which again is a contradiction.

For $j \geqq 2$, let us first suppose $d>8$. Again $a_{\lambda+2}=d+5, a_{\lambda+3}=d+6$. If $j=2$, we have $a_{\lambda+4}=2^{\lambda}=d+8, a_{\lambda+5}=d+10$ and comparing the coefficients of $x^{2 d+14}$ we find it to be 2 or 3 on the left-hand side, since the term $x^{2 d+14}$ is obtained as $x^{3} \cdot x^{5+d} \cdot x^{6+d}$ and as $x^{4+d} \cdot x^{10+d}$ and $2^{\lambda+1} \neq 2 d+14$, while the coefficient of the corresponding term on the right-hand side is 1 in view of the fact that $3 d+6>2 d+14$ for $d>8$. If $j=3: a_{\lambda+4}=2^{\lambda}=d+9, a_{\lambda+5}=d+10$, so that again we obtain a contradiction comparing the coefficient of $x^{2 d+14}$. For $j \geqq 4$, we have $a_{\lambda+4}=d+10$, so that again comparing the coefficient of $x^{2 d+14}$ gives us the desired conclusion.

For $d \leqq 8$ contradictions are easily obtained by considering the cases individually: for $d=5$ and 6 by comparing the coefficient of $x^{16}$, for $d=7$ by comparing the coefficient of $x^{30}$ and for $d=8$ by comparing the coefficient of $x^{27}$.

4. Proof of Theorem 3. Suppose again that the theorem is false and that there exists such a set of integers $a_{1}<a_{2}<a_{3}<\cdots$, then

$$
\begin{aligned}
\prod_{\nu=1}^{\infty}\left(1-x^{a_{\nu}}\right)^{-1}= & \sum_{s=0}^{\infty} \frac{x^{s+d s(s-1) / 2}}{(1-x)\left(1-x^{2}\right) \cdots\left(1-x^{s}\right)} \\
& -\sum_{t=0}^{\infty} A(t) x^{t},
\end{aligned}
$$

where $A(t)$ denotes the number of partitions of $t$ into parts differing by at least $d$ and each partition is such that it contains at least two consecutive multiples of $d$, that is,

$$
A(t)=0
$$

for $t=0,1,2, \cdots, 3 d-1, A(3 d)=1$. Since $a_{1}=1$, we multiply (20) by $1-x$ and obtain:

$$
\begin{aligned}
\prod_{\nu=2}^{\infty}\left(1-x^{a_{\nu}}\right)^{-1}=1 & +\frac{x^{d+2}}{1-x^{2}}+\frac{x^{3 d+3}}{\left(1-x^{2}\right)\left(1-x^{3}\right)}+\cdots \\
& -\sum_{t=3 d}^{\infty} A(t) x^{t}+\sum_{t=3 d}^{\infty} A(t) x^{t+1}
\end{aligned}
$$

Now $a_{2}=d+2$ and we multiply (21) by $1-x^{d+2}$ : 


$$
\begin{aligned}
\prod_{\nu=3}^{\infty}\left(1-x^{a}\right)^{-1}=1 & -x^{d+2}+\frac{x^{d+2}}{1-x^{2}}-\frac{x^{2 d+4}}{1-x^{2}} \\
& +\frac{x^{3 d+3}}{\left(1-x^{2}\right)\left(1-x^{3}\right)}+\cdots \\
& -\sum_{t=3 d}^{\infty} A(t) x^{t}\left(1-x^{d+2}\right) \\
& +\sum_{t=3 d}^{\infty} A(t) x^{t+1}\left(1-x^{d+2}\right) .
\end{aligned}
$$

If $d$ is odd, the coefficient of $x^{2 d+4}$ on the right-hand side of (22) is equal to -1 as $3 d+1>2 d+4$ for $d>3$, which is a contradiction of the fact that the corresponding term on the left-hand side has a nonnegative coefficient. If $d$ is even, the coefficient of $x^{3 d}$ on the righthand side is -1 and non-negative on the left-hand side, which completes the proof of the theorem.

The above can be extended to more general cases by similar procedure. It can, for example, be shown that there cannot exist a dual to Schur's theorem in the sense that the second of the RogersRamanujan identities is a dual to the first one, that is, that the number of partitions of $n$ into parts differing by at least 3, no part being equal to 1 , where parts divisible by 3 differ by at least 6 , is not equal to the number of partitions of $n$ into parts taken from any set of integers whatsoever.

5. Proof of Theorem 4. In case the theorem were false we would have, recalling (2):

$$
\begin{aligned}
\sum_{t=0}^{\infty}\left(x^{a_{1}}+x^{a_{2}}+\cdots\right)^{t} & =\sum_{s=0}^{\infty} \frac{s ! x^{m s+d s(s-1) / 2}}{(1-x)\left(1-x^{2}\right) \cdots\left(1-x^{s}\right)} \\
& =1+\frac{x^{m}}{1-x}+\frac{2 x^{2 m+d}}{(1-x)\left(1-x^{2}\right)}+\cdots .
\end{aligned}
$$

Therefore $a_{1}=m$; then (23) becomes

$$
\begin{aligned}
\frac{1}{1-x^{m}}+x^{a_{2}}+x^{a_{3}} & +\cdots \\
& =1+\frac{x^{m}}{1-x}+\frac{2 x^{2 m+d}}{(1-x)\left(1-x^{2}\right)}+\cdots .
\end{aligned}
$$

If $m=1$, we obtain: 


$$
x^{a_{2}}+x^{a_{3}}+\cdots=\frac{2 x^{2+d}}{(1-x)\left(1-x^{2}\right)}+\cdots,
$$

which is a contradiction, as the coefficient of the term with smallest exponent on the left-hand side of (25) is equal to 1 , whereas it is equal to 2 on the right-hand side.

If $m>1$, we multiply (24) by $1-x^{m}$ and obtain:

$$
\begin{aligned}
& 1+\left(x^{a_{2}}+x^{a_{3}}+\cdots\right)\left(1-x^{m}\right) \\
& =1+x^{m+1}+x^{m+2}+\cdots+x^{2 m-1}+\frac{2 x^{2 m+d}\left(1-x^{m}\right)}{(1-x)\left(1-x^{2}\right)}+\cdots,
\end{aligned}
$$

so that $a_{2}=m+1$.

Now if $d>1$, the coefficient of $x^{2 m+1}$ on the right-hand side of (26) equals 0 , while we find the coefficient of the same term on the lefthand side to be greater than or equal to 1 , which is evident, if we recall (23). For $d=1$ a contradiction is easily obtained by considering more terms in (24).

The preceding shows in particular that there is no analog in the theory of compositions to the Rogers-Ramanujan identities and to Euler's Theorem.

The University of California 